\title{
Frequency-Domain Circuit Model and Analysis of Coupled Magnetic Resonance Systems
}

\author{
Jin Huh ${ }^{\dagger}$, Wooyoung Lee ${ }^{*}$, Suyong Choi ${ }^{* *}$, Gyuhyeong Cho ${ }^{*}$, and Chuntaek Rim ${ }^{* *}$ \\ $\dagger^{* *}$ Dept. of Electrical Engineering, KAIST, Daejeon, Korea \\ ${ }^{* *}$ Dept. of Nuclear \& Quantum Engineering, KAIST, Daejeon, Korea
}

\begin{abstract}
An explicit frequency-domain circuit model for the conventional coupled magnetic resonance system (CMRS) is newly proposed in this paper. Detail circuit parameters such as the leakage inductances, magnetizing inductances, turn-ratios, internal coil resistances, and source/load resistances are explicitly included in the model. Accurate overall system efficiency, DC gain, and key design parameters are deduced from the model in closed form equations, which were not available in previous works. It has been found that the CMRS can be simply described by an equivalent voltage source, resistances, and ideal transformers when it is resonated to a specified frequency in the steady state. It has been identified that the voltage gain of the CMRS was saturated to a specific value although the source side or the load side coils were strongly coupled. The phase differences between adjacent coils were $\pi / 2$, which should be considered for the EMF cancellations. The analysis results were verified by simulations and experiments. A detailed circuit-parameter-based model was verified by experiments for $500 \mathrm{kHz}$ by using a new experimental kit with a class-E inverter. The experiments showed a transfer of $1.38 \mathrm{~W}$ and a $40 \%$ coil to coil efficiency.
\end{abstract}

Key words: Coupled magnetic resonance system, Inductive power transfer, Magnetic resonance, Wireless power transfer system

\section{NOMENCLATURE}

$L_{l S}$ : leakage inductance of a source coil

$L_{l T}$ : leakage inductance of a transmitter coil

$L_{l R}$ : leakage inductance of a receiver coil

$L_{I L}$ : leakage inductance of a load coil

$L_{m s}$ : magnetizing inductance of a source coil

$L_{m T}$ : magnetizing inductance of a transmitter coil

$L_{m R}$ : magnetizing inductance of a receiver coil

$L_{m L}:$ magnetizing inductance of a load coil

$C_{S}$ : compensation capacitance of a source coil

$C_{T}$ : compensation capacitance of a transmitter coil

$C_{R}$ : compensation capacitance of a receiver coil

$C_{L}$ : compensation capacitance of a load coil

$r_{S}$ : internal resistance of a source coil

$r_{T}$ : internal resistance of a transmitter coil

$r_{R}$ : internal resistance of a receiver coil

Manuscript received Aug. 10, 2011; revised Jan. 21, 2013

Recommended for publication by Associate Editor Tae-Woong Kim.

†Corresponding Author: hk221@kaist.ac.kr

Tel: +82- 52-259-1282, KAIST

${ }^{*}$ Dept. of Electrical Engineering, KAIST, Korea

${ }^{* *}$ Dept. of Nuclear \& Quantum Engineering, KAIST, Korea
$r_{L}$ : internal resistance of a load coil

$R_{\text {in }}$ : internal resistance of a voltage source

$R_{L}:$ load resistance

$R_{S}:$ sum of $R_{\text {in }}$ and $r_{S}$

$R_{e S T}$ : equivalent resistance from a source coil to a transmitter coil

$R_{e L R}$ : equivalent resistance from a load coil to a receiver coil

$R_{e S R}$ : equivalent resistance from a source coil to a receiver coil

$R_{e L T}$ : equivalent resistance from a load coil to a transmitter coil

$R_{e S L}$ : equivalent resistance from a source coil to a load coil

$R_{e L S}$ : equivalent resistance from a load coil to a source coil

$V_{T t h}$ : Thevenin equivalent voltage of a transmitter coil

$V_{R t h}$ : Thevenin equivalent voltage of a receiver coil

$V_{L t h}:$ Thevenin equivalent voltage of a load coil

$\omega_{s}:$ source angular frequency

$G_{V 0}$ : open circuit voltage gain between a source and a load without $R_{L}$

$G_{V}$ : voltage gain between a source and a load with $R_{L}$

\section{INTRODUCTION}

As mobile electronic devices and robots have evolved and 
become widely used regardless of the time and place, it becomes a very important issue to resolve the "power hungry problem" by freely charging devices anytime and anywhere. To solve this problem, wireless power transfer technologies have been proposed [1]-[9]. However, these technologies, which are based on inductive coupling, have been limited to the proximity since the transferred power is drastically reduced over large distances. In 2007, an innovative wireless power transfer system, the coupled magnetic resonance system (CMRS), was proposed [10]. This system also uses magnetic fields, but it has two more coils, a transmitter coil and a receiver coil, which are fully resonated at a specific resonant frequency. This system can light up a $60 \mathrm{~W}$ light bulb, which is placed over $2 \mathrm{~m}$ away from the power source, with about a $45 \%$ coil-to-coil efficiency. After this work was presented, many similar studies have been conducted by several researchers [11]-[14]. Intel demonstrated another CMRS called a wireless resonant energy link (WREL) [11]. Sony Corp. also announced a CMRS capable of a $60 \mathrm{~W}$ transfer over a distance of $50 \mathrm{~cm}$ with a coil-to-coil efficiency of $80 \%$ [12]. The Electronics and Telecommunications Research Institute (ETRI) also presented experimental results for its CMRS by lighting LED lamps [13].

In previous studies [10], [11], the equivalent models for the CMRS, which are the coupled mode theory [10] and scattering parameters [11], are very complicated. They are not quite useful for practical designs because theoretical parameters such as the coupling factor and the impedance matching concept were used without using lumped elements. As a result, the analyses can hardly be conducted without the aid of computers. They cannot clearly show what parameters limit the power transfer due to a lack of explicit closed form design equations. Furthermore, they operate at extremely large quality factor, Q $(\sim 2,000)$ and a high resonant frequency $(\sim 13.56 \mathrm{MHz})$. Hence it has been a difficult problem to design appropriate coils for these CMRS. Closed form analysis results for coupled resonators have been proposed [14]. However, it was conducted only for two resonant coils, the source and the load coils. Therefore, the analysis results could not explain a CMRS where three coupled resonators exist.

In this paper, an explicit equivalent circuit model of a CMRS, which includes all of the detail parameters for four coils and three magnetic couplings, is proposed. By applying an appropriate full resonance scheme, the CMRS is simply modeled as an equivalent voltage source with resistors regardless of its complexity. The analysis results of the proposed model are verified by simulations and experiments with good agreements.

\section{Proposed Circuit Model AND Static ANALYSIS}

\section{A. Simplified Equation Circuit of the CMRS}

The modeled CMRS is composed of a power source, four coils, four resonant capacitors, and a load, as shown in Fig. 1. The power source can be either a switching inverter or an analog amplifier. The capacitors for the transmitter and receiver coils, i.e., $C_{T}$ and $C_{R}$ can be either parasitic or lumped. For a complete and detail description of the CMRS, the coupling transformers in Fig. 1 are fully resolved by minute circuit elements including ideal transformers, as shown in Fig. 2. In other words, each coil is composed of a leakage inductance $L_{l}$, a magnetizing inductance $L_{m}$, an internal resistance $r$, and an ideal transformer. The weak couplings between the source and receiver coils, the source and load coils, and the transmitter and load coils are neglected from consideration for the sake of a convenient analysis. Under the condition of using the lumped compensation capacitor, the circuit parameters shown in Fig. 2 represent the sum of all of the serial components, e.g., $L_{l T}$ represents the sum of the source side secondary leakage inductance and the transmitter side primary leakage inductance. For circuit symmetry, $L_{m T}$ is intentionally split into two. The proposed equivalent circuit is now simplified by applying the fully resonance scheme [6] to all four resonant circuits at the source angular frequency $\omega_{s}$, respectively.

Fig. 3 shows the circuit simplification of the series-series resonant circuit, where the capacitances of $C_{1}$ and $C_{2}$ satisfy the fully-resonant conditions of (1) and (2), respectively.

$$
j \omega_{s} L_{m x}+\frac{j \omega_{s} L_{l 2}}{n^{2}}+\frac{1}{j \omega_{s} n^{2} C_{2}}=0
$$

Under the fully-resonant condition, by applying (1), the parallel circuit of $L_{m x}$ and the secondary impedance reflected to the primary, as shown in Fig. 3 (b), are simplified to $L_{m x}$ and its equivalent resistance $n^{2} \omega_{s}^{2} L_{m x}^{2} / R_{2}$, as shown in Fig. 3 (c).

$$
j \omega_{s} L_{m x}+j \omega_{s} L_{l 1}+\frac{1}{j \omega_{s} C_{1}}=0
$$

By applying (2) again, Fig. 3 (c) can be simplified to Fig. 3 (d), which is composed of only pure resistors. $x$ denotes an arbitrary stage, i.e. the source, transmitter, receiver, or load stage.

By applying the same approach to the CMRS, it is equivalently transformed from the load coil to the source coil and simplified step by step, as shown in Fig. 4 (a)-(e). Fig. 5 shows the equivalently simplified circuits of the CMRS transformed to each coil, respectively. Under the fully-resonant condition for the CMRS, the equivalent resistance transformed to each coil of the source, transmitter, receiver, and load can be given in (3) - (8). 


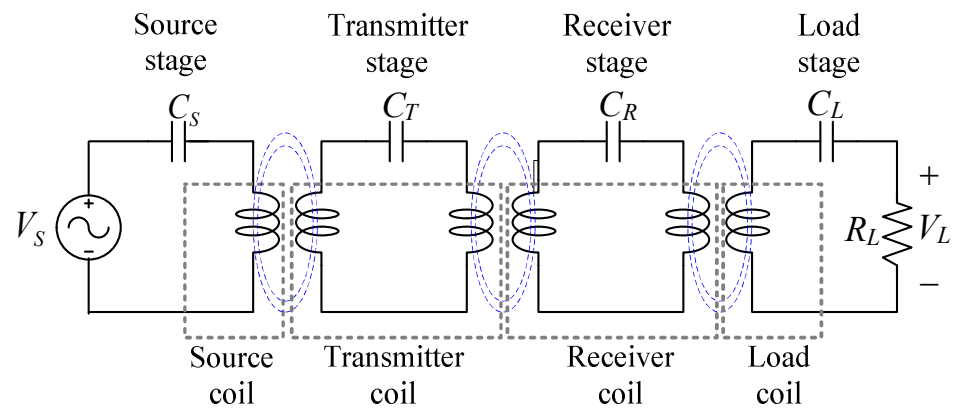

Fig. 1. Overall configuration of the coupled magnetic resonance system (CMRS) showing magnetic coupling.

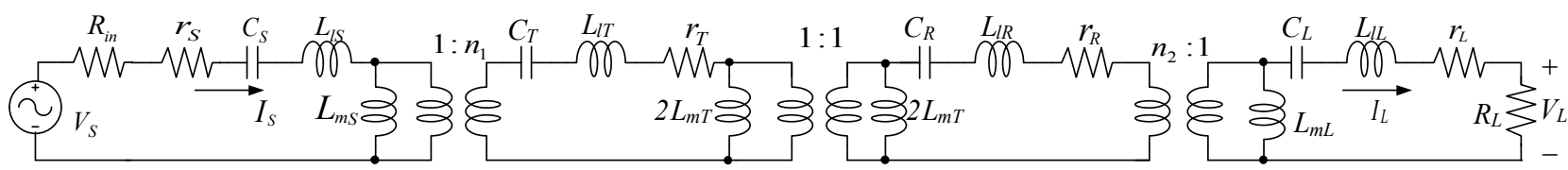

Fig. 2. Proposed explicit circuit model with all circuit parameters included showing symmetry.

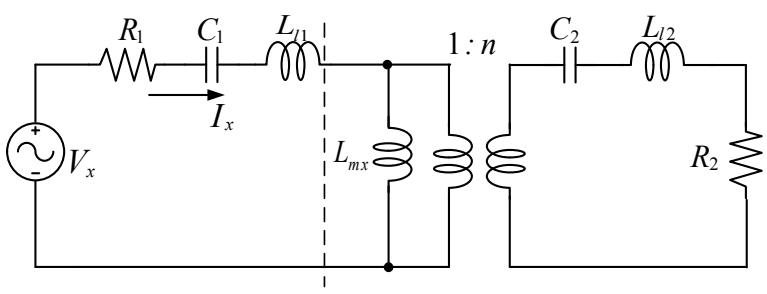

(a) Equivalent circuit of series-series resonant circuit.

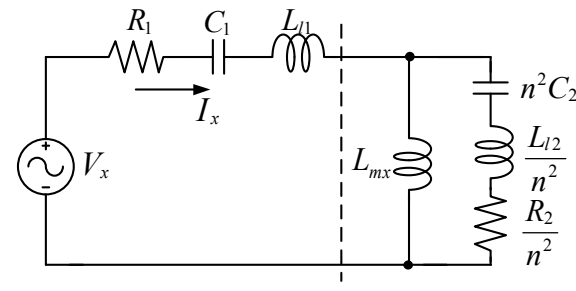

(b) Equivalent circuit from the source side.

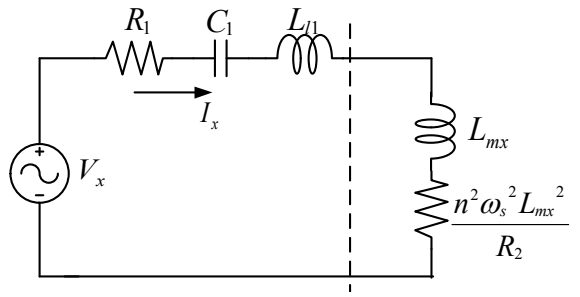

(c) Equivalent circuit simplified by applying (1).

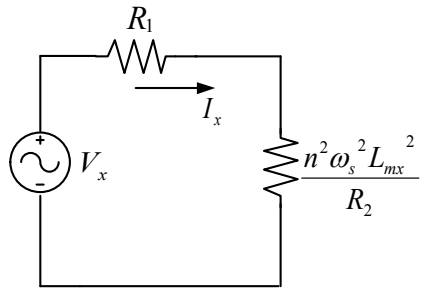

(d) Equivalent circuit simplified by applying (2).

Fig. 3. An example of simplification process for a series-series resonant transformer circuit from the source side.

$$
\begin{gathered}
R_{e S T}=\frac{\left(n_{1} \omega_{s} L_{m S}\right)^{2}}{r_{S}+R_{i n}}=\frac{\left(n_{1} \omega_{s} L_{m S}\right)^{2}}{R_{S}} \\
R_{e L R}=\frac{\left(n_{2} \omega_{s} L_{m L}\right)^{2}}{R_{L}+r_{L}} \\
R_{e S R}=\frac{\left(\omega_{s} L_{m T}\right)^{2}}{r_{T}+\frac{\left(n_{1} \omega_{s} L_{m S}\right)^{2}}{R_{S}}}=\frac{R_{S}\left(\omega_{s} L_{m T}\right)^{2}}{r_{T} R_{S}+\left(n_{1} \omega_{s} L_{m S}\right)^{2}} \\
R_{e L T}=\frac{\left(\omega_{s} L_{m T}\right)^{2}}{r_{R}+\frac{\left(n_{2} \omega_{s} L_{m L}\right)^{2}}{r_{L}+R_{L}}}=\frac{\left(r_{L}+R_{L}\right)\left(\omega_{s} L_{m T}\right)^{2}}{r_{R}\left(r_{L}+R_{L}\right)+\left(n_{2} \omega_{s} L_{m L}\right)^{2}}
\end{gathered}
$$

$$
\begin{gathered}
R_{e S L}=\frac{\left(n_{2} \omega_{s} L_{m L}\right)^{2}\left\{r_{T} R_{S}+\left(n_{1} \omega_{s} L_{m S}\right)^{2}\right\}}{r_{R}\left\{r_{T} R_{S}+\left(n_{1} \omega_{s} L_{m S}\right)^{2}\right\}+R_{S}\left(\omega_{s} L_{m T}\right)^{2}} \\
R_{e L S}=\frac{\left(n_{1} \omega_{s} L_{m S}\right)^{2}\left\{r_{R}\left(r_{L}+R_{L}\right)+\left(n_{2} \omega_{s} L_{m L}\right)^{2}\right\}}{r_{T}\left\{r_{R}\left(r_{L}+R_{L}\right)+\left(n_{2} \omega_{s} L_{m L}\right)^{2}\right\}+\left(\omega_{s} L_{m T}\right)^{2}\left(r_{L}+R_{L}\right)}(8)
\end{gathered}
$$

The equivalent voltage transformed to each coil of the source, transmitter, receiver, and load can be given in (9) - (11).

$$
\begin{gathered}
V_{T t h}=\frac{j n_{1} \omega_{s} L_{m S}}{R_{S}} V_{S} \\
V_{R t h}=\frac{j \omega_{s} L_{m T}}{r_{T}+R_{e S T}} V_{T t h}=\frac{-n_{1} \omega_{s}^{2} L_{m S} L_{m T}}{n_{1}^{2} \omega_{s}{ }^{2} L_{m S}{ }^{2}+r_{T} R_{S}} V_{S}
\end{gathered}
$$




$$
V_{L t h}=\frac{j \omega_{s} L_{m L}}{r_{R}+R_{e S R}} V_{R t h}=\frac{-j n_{1} n_{2} \omega_{s}^{3} L_{m S} L_{m T} L_{m L}}{\left\{r_{T} R_{S}+\left(n_{1} \omega_{s} L_{m S}\right)^{2}\right\}\left\{r_{R}+\frac{R_{S}\left(\omega_{s} L_{m T}\right)^{2}}{r_{T} R_{S}+\left(n_{1} \omega_{s} L_{m S}\right)^{2}}\right\}} V_{S}
$$

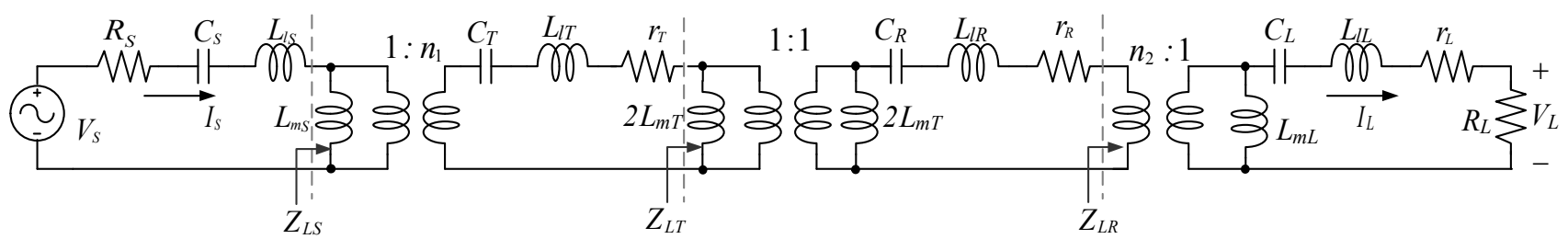

(a) Original proposed explicit circuit model.

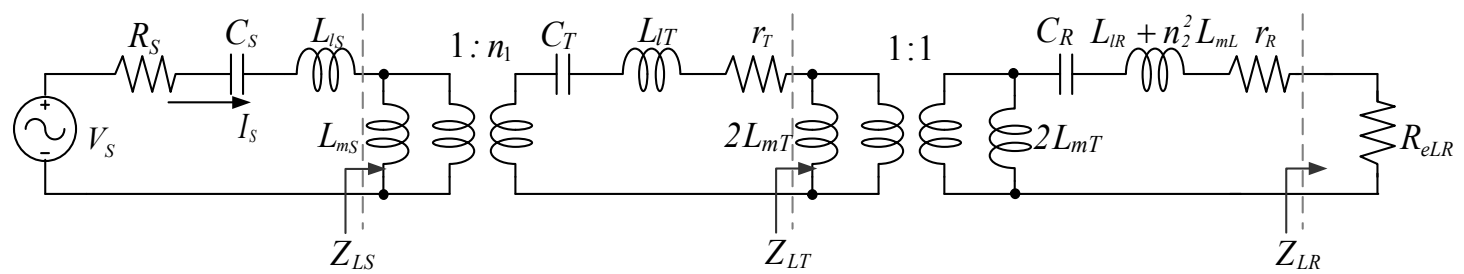

(b) Simplified circuit with $Z_{L R}$.

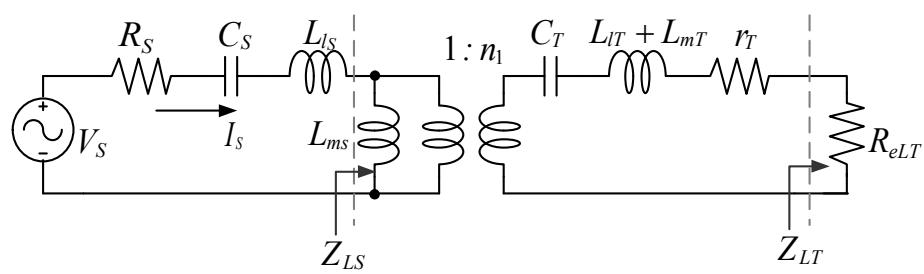

(c) Simplified circuit with $Z_{L T}$.

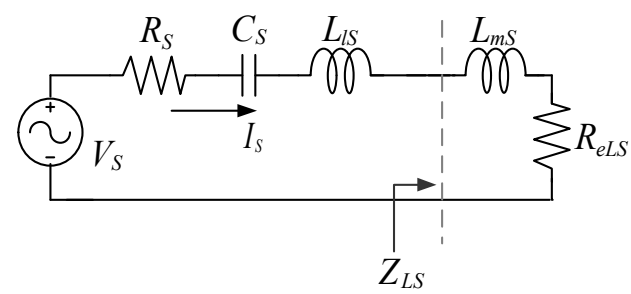

(d) Simplified circuit with $Z_{L S}$.

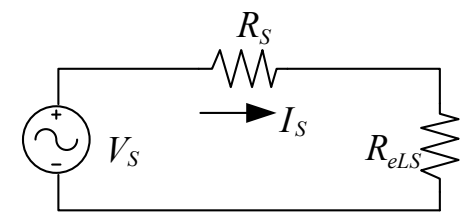

(e) Simplified circuit transformed to the source coil.

Fig. 4. Equivalent circuit transformation from the load coil to the source coil. 

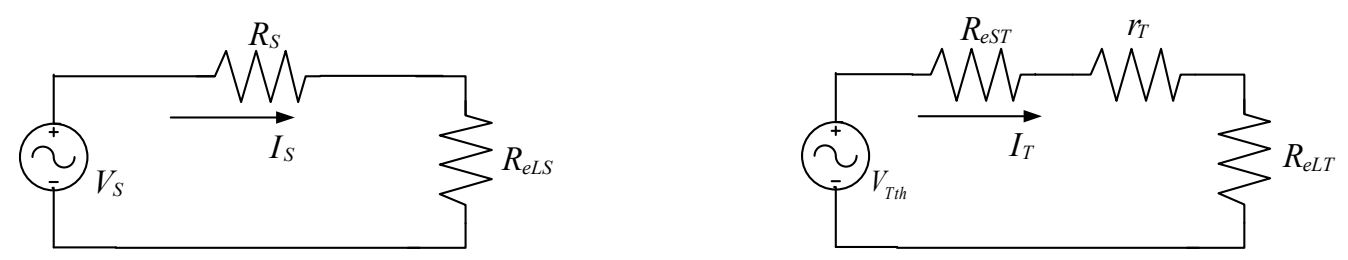

(a) Equivalently simplified circuit transformed to the source coil. (b) Equivalently simplified circuit transformed to the transmitter coil.
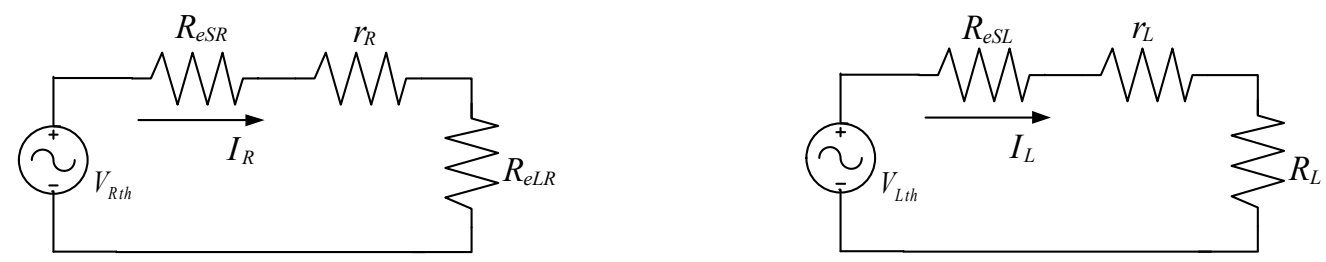

(c) Equivalently simplified circuit transformed to the receiver coil. (d) Equivalently simplified circuit transformed to the load coil.

Fig. 5. Simplified circuits of the CMRS in the fully-resonant conditions.

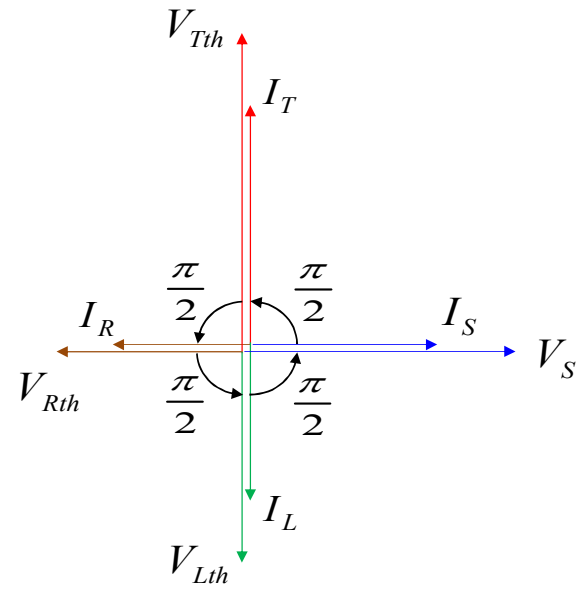

Fig. 6. Phasor vector plots of all coils.

The current flowing through the each coil, as shown in Fig. 5 , can also be obtained by using the equivalently transformed resistance and voltage given in (3)-(11). Under the fully-resonant condition for the CMRS, the open circuit voltage gain $G_{V o}$ between the source voltage $V_{S}$ and the induced voltage of the load coil $V_{L t h}$ for the no load condition can also be found from (11) and given in (12). The overall system voltage gain from the source coil to the load coil is given in (13). From the equivalent circuits in Fig. 5 and the Thevenin equivalent voltages given from (9) to (11), it has been verified that the phases of the voltage and current in each coil were in-phase because each coil had only pure resistance when each coil was fully resonated. However, the phases between adjacent coils were found to be sequentially different by $\pi / 2$ since the resonant current built up the induced voltage at the magnetizing inductance of the adjacent coil by $\pi / 2$. The $-j$ sign, which appears in (11), was the result of three successive phase shifts, as shown in Fig. 6. These phase relationships should be considered for lowering the EMF levels. As the phase of the current in each coil differs by $\pi / 2$, an EMF cancellation scheme should be employed for each coil. In other words, there is no simple way of cancelling the EMF generated from four coils by an EMF cancel coil, for example.

$$
\begin{gathered}
G_{V o}=\left|\frac{V_{L t h}}{V_{s}}\right|=\frac{n_{1} n_{2} \omega_{s}^{3} L_{m S} L_{m T} L_{m L}}{\left\{r_{T} R_{S}+\left(n_{1} \omega_{s} L_{m S}\right)^{2}\right\}\left\{r_{R}+\frac{R_{S}\left(\omega_{s} L_{m T}\right)^{2}}{r_{T} R_{S}+\left(n_{1} \omega_{s} L_{m S}\right)^{2}}\right\}} \\
G_{V}=\left|\frac{V_{L}}{V_{s}}\right|=G_{V o} \frac{R_{L}}{R_{e S L}+r_{L}+R_{L}}=\frac{n_{1} n_{2} \omega_{s}^{3} L_{m S} L_{m T} L_{m L}}{\left\{r_{T} R_{S}+\left(n_{1} \omega_{s} L_{m S}\right)^{2}\right\}\left\{r_{R}+\frac{R_{S}\left(\omega_{s} L_{m T}\right)^{2}}{r_{T} R_{S}+\left(n_{1} \omega_{s} L_{m S}\right)^{2}}\right\}} \frac{R_{L}}{R_{e S L}+r_{L}+R_{L}}
\end{gathered}
$$




\section{B. Further Simplification of a Symmetric Circuit}

When all of the internal resistances are identical and the circuit parameters are symmetric as given in (14), (12) is drastically simplified to (15).

$$
\begin{gathered}
r_{S}=r_{T}=r_{R}=r_{L} \equiv r, L_{m S}=L_{m L} \equiv L_{m}, n_{1}=n_{2} \equiv n \\
R_{e q} \equiv \frac{\left(n \omega_{s} L_{m}\right)^{2}}{R_{S}} \\
G_{V o}=\frac{\omega_{s} L_{m T} R_{e q}}{r\left(r+R_{e q}\right)+\left(\omega_{s} L_{m T}\right)^{2}}
\end{gathered}
$$

The open circuit voltage gain $G_{V o}$ of (15) increases as $R_{e q}$ or $L_{m}$, when $n$ increase, as shown in (14), but decreases when $R_{S}$ increases.

By applying the same condition of (14) to the equivalent resistances $R_{e S L}$ and $R_{e L S}$, given in (7) and (8), they are rewritten as follows:

$$
\begin{gathered}
R_{e S L}=\frac{R_{S} R_{e q}\left(r+R_{e q}\right)}{r\left(r+R_{e q}\right)+\left(\omega_{s} L_{m T}\right)^{2}} \\
R_{e L S}=\frac{R_{S} R_{e q}\left(r+\frac{R_{S} R_{e q}}{r+R_{L}}\right)}{r\left(r+\frac{R_{S} R_{e q}}{r+R_{L}}\right)+\left(\omega_{s} L_{m T}\right)^{2}}
\end{gathered}
$$

\section{Ideal No Loss Case for the Symmetric Circuit}

For an ideal no loss case for the symmetric circuit, $r$ in (14) becomes 0 but $R_{S}$ remains non-zero. Therefore, (15) - (17) can be further simplified in terms of $G_{V o}$ as follows:

$$
\begin{aligned}
G_{V o} & =\frac{R_{e q}}{\omega_{s} L_{m T}}=\frac{\omega_{s} n^{2} L_{m}^{2}}{R_{S} L_{m T}} \\
R_{e S L} & =\frac{R_{S} R_{e q}{ }^{2}}{\left(\omega_{s} L_{m T}\right)^{2}}=R_{S} G_{V o}^{2} \\
R_{e L S} & =\frac{\left(R_{S} R_{e q}\right)^{2}}{R_{L}\left(\omega_{s} L_{m T}\right)^{2}}=\frac{R_{S}^{2}}{R_{L}} G_{V o}^{2}
\end{aligned}
$$

The source and load currents are found from Figs. 4 and 5, and (13) as follows:

$$
\begin{gathered}
I_{S}=\frac{V_{S}}{R_{S}+R_{e L S}} \\
I_{L}=\frac{G_{V o} V_{S}}{R_{L}+R_{e S L}}
\end{gathered}
$$

By using (21), (22) and (18), the power efficiency of the CMRS is determined as follows:

$$
\begin{aligned}
\eta & =\frac{P_{L}}{P_{S}}=\frac{\left|I_{L}\right|^{2} R_{L}}{\left|V_{S}\right|\left|I_{S}\right|}=\frac{R_{L}\left(R_{S}+R_{e L S}\right) G_{V o}^{2}}{\left(R_{L}+R_{e S L}\right)^{2}} \\
& =\frac{R_{L}\left(R_{S}+R_{S}^{2} G_{V o}^{2} / R_{L}\right) G_{V o}^{2}}{\left(R_{L}+R_{S} G_{V o}^{2}\right)^{2}}=\frac{R_{S} G_{V o}^{2}}{R_{L}+R_{S} G_{V o}^{2}} \\
& =\frac{1}{1+R_{L} /\left(R_{S} G_{V o}^{2}\right)}=\frac{1}{1+R_{L} R_{S} L_{m T}^{2} /\left(\omega_{s}^{2} n^{4} L_{m}^{4}\right)}
\end{aligned}
$$

From (23), the load resistance $R_{L}$ as well as the source resistance $R_{S}$ should be small for a high power efficiency.

On the other hand, the output power $P_{L}$ can be obtained from (13), (18), and (19) as follows:

From (24), it can be seen that the output power decreases as the source resistance $R_{S}$ increases, and an optimum source angular frequency $\omega_{s}$ exists for maximum power delivery. It can also be seen that an optimum load resistance $R_{L}$ exists for maximum power delivery as follows:

$$
\begin{gathered}
P_{L}=\frac{\left|V_{L}\right|^{2}}{R_{L}}=\left(\frac{G_{V o} R_{L}\left|V_{S}\right|}{R_{L}+R_{e S L}}\right)^{2} \frac{1}{R_{L}}=\left(\frac{G_{V o}\left|V_{S}\right|}{R_{L}+R_{S} G_{V o}^{2}}\right)^{2} R_{L} \\
=\frac{R_{L}\left|V_{S}\right|^{2}}{\left(R_{L} G_{V o}^{-1}+R_{S} G_{V o}\right)^{2}}=\frac{R_{L}\left|V_{S}\right|^{2}}{\left(\frac{R_{S} R_{L} L_{m T}}{\omega_{s} n^{2} L_{m}^{2}}+\frac{\omega_{s} n^{2} L_{m}^{2}}{L_{m T}}\right)^{2}} \\
\frac{\partial P_{L}}{\partial R_{L}}=0 \Rightarrow R_{L}=G_{V o}^{2} R_{S}
\end{gathered}
$$

Applying (25) to (24) results in the maximum output power as follows:

$$
P_{L, \text { max }}=\left.\frac{R_{L}\left|V_{S}\right|^{2}}{\left(R_{L} G_{V o}^{-1}+R_{S} G_{V o}\right)^{2}}\right|_{R_{L}=G_{V o}^{2} R_{S}}=\frac{\left|V_{S}\right|^{2}}{4 R_{S}}
$$

It can be seen from (26) that the source resistance is the ultimate limiting factor for the maximum power delivery as long as the power ratings of all the coils and capacitors are sufficiently high.

When $R_{S}$ is zero, (24) becomes the following:

$$
\left.P_{L}\right|_{R_{S}=0}=\left(\frac{L_{m T}\left|V_{S}\right|}{\omega_{s} n^{2} L_{m}^{2}}\right)^{2} R_{L}
$$

It can be seen from (27) that an ideal CMRS can provide more power for a large $R_{L}$ and small $\omega_{s}$. However, the effect of the other parameters in (27) on the output power are not straightforward. For the given physical dimensions, $L_{m T}$ is proportional to $n^{2}$. Hence its effect on the output power is negligible. 


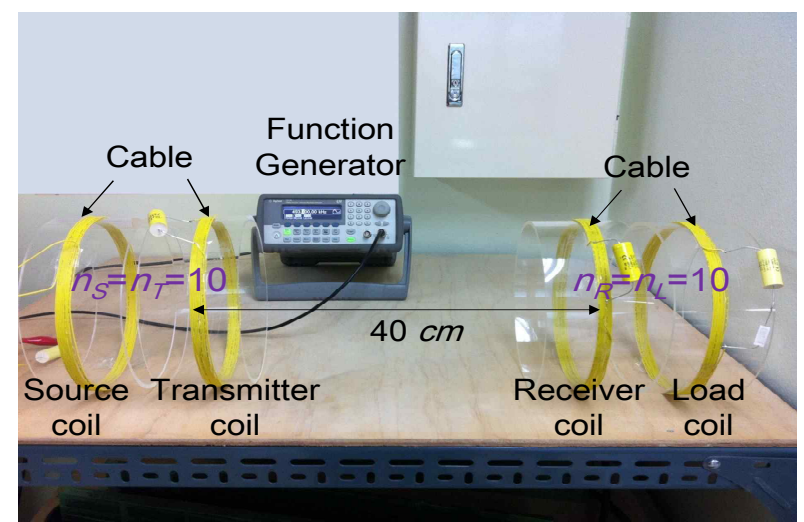

Fig. 7. The CMRS for experiments.

D. Ideal No Loss Case for the Symmetric Circuit with $R_{S}=$ $R_{L}$

For a special condition where $R_{S}=R_{L},(20)$ can be rewritten as (28). Thus the equivalent resistances $R_{e S L}$ and $R_{e L S}$ become the same.

$$
R_{e L S}=\frac{R_{S}^{2}}{R_{L}} G_{V o}^{2}=R_{S} G_{V o}^{2}=R_{e S L}
$$

Under this condition, the efficiency $\eta$ of (23) and the output power $P_{L}$ of (24) are simplified, respectively.

$$
\begin{aligned}
\left.\eta\right|_{R_{S}=R_{L}} & =\frac{G_{V o}{ }^{2}}{1+G_{V o}^{2}} \\
\left.P_{L}\right|_{R_{S}=R_{L}} & =\left(\frac{G_{V o}}{1+G_{V o}^{2}}\right)^{2} \frac{\left|V_{S}\right|^{2}}{R_{L}}
\end{aligned}
$$

It can be seen from (29) and (30) that a larger power efficiency is obtained for a larger $G_{V o}$. However, the maximum output power is obtained at $G_{V_{o}}=1$. Therefore, it can be concluded that the output power and the power efficiency should have a trade-off.

\section{SIMULATION AND EXPERIMENTAL VERIFICATIONS}

The experimental kit is shown in Fig. 7, where the radius of all the coils was $10.5 \mathrm{~cm}$, and the number of turns for each coil was 10 . The distance between the transmitter coil and the receiver coil was $40 \mathrm{~cm}$. The magnetizing inductances, $L_{m s}$ and $L_{m L}$, were determined to satisfy the maximum power transfer conditions, $R_{S}=R_{e L S}$ and $R_{L}=R_{e S L}$. The measured parameters for the leakage inductances, magnetizing inductances, and internal resistances of the coils are listed in Table 1. The compensation capacitances of each coil for satisfying the fully-resonant condition were calculated from the extracted parameters and connected to each coil. The input resistance of the function generator was $50 \Omega$, so load resistance was determined to be the same value of $50 \Omega$. The function generator output was about $12 \mathrm{~V}$ in the no load condition, which meant all of the coils were open. However, it became about half when the CMRS properly operated at the fully-resonant frequency of $493.3 \mathrm{kHz}$.

By using the measured parameters of the CMRS experiments in Table 1, it was verified by PSIM simulations that the theoretical predictions of (15), (17), (23), and (24) were good with their simulation errors of less than $1 \%$. The simulation result of the equivalent resistance $R_{e S L}$ under the condition of (16) is shown in Fig. 8 (a). The equivalently transformed resistance $R_{e L S}$ or $R_{e S L}$ is highly dependent on the magnetizing inductance between adjacent coils such as $L_{m S}$ and $L_{m L}$ and it continuously increases as the magnetizing inductance increases. This is because the equivalently transformed resistance is proportional to the square of the product of the magnetizing inductance and the resonant frequency. In addition, the internal resistances of each coil also affect the equivalently transformed resistance because the transformed resistance is inversely proportional to the internal resistance as given in (3)-(8). Due to these high transformed resistances, the output power of the CMRS is limited.

The voltage gain of the CMRS increases as $L_{m S}$ increases, as shown in Fig. 8 (b). By determining the optimum value of $L_{m S}, 2.1 \mu \mathrm{H}$, where the equivalently transformed resistance from the load to the source $R_{e L S}$ is the same as the internal resistance of the voltage source $R_{S}$, the maximum power was achieved, as shown in Fig. 8 (c).

Fig. 8 (c) and Fig. 8 (d) also show that the internal resistance of each coil lowers the output power and the power efficiency. To get a high power and efficiency, the internal resistance should be sufficiently low. For example, the power efficiency was about $61 \%$ when $r=0.1 \Omega$, but it increased up to $90 \%$ when $r=0.01 \Omega$, as shown in Fig. 8 (d).

It has been verified by experiments that the phase of the voltage induced by each coil is consecutively different by $\pi / 2$ and that the source voltage leads the output voltage by an expected $\pi / 2$, as shown in Fig. 9. It has also been verified that the phase of the voltage and the current of each coil are same, as shown in Fig. 9. This is because there are only resistive components in the fully-resonant condition.

The voltage gain and the load power along the load resistance were also measured and compared with the theoretical prediction, as shown in Fig. 10. The voltage gain between the source and the load was increased as the load resistance increased, and it converged to 0.247 in measurement and 0.276 in calculation, as shown in Fig. 10 (a). When the CMRS was fully resonated, the measured output voltage was about $1.7 \mathrm{~V}$, which was almost the same as the calculated load voltage of $1.67 \mathrm{~V}$. In addition, it decreased from the maximum value as the operating frequency deviated from the resonant frequency, as shown in Fig. 10 (b). The maximum power was achieved when the load resistance was the same as the internal resistance of the voltage source, as shown in Fig. 10 (c). 


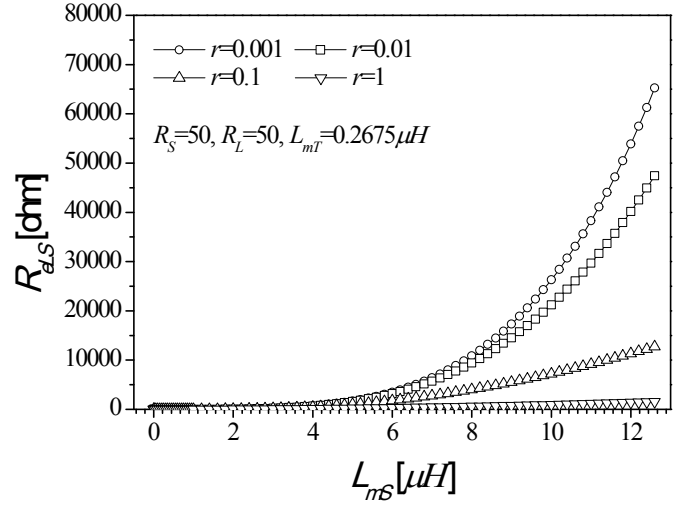

(a) Equivalent resistance from the load coil to the source coil

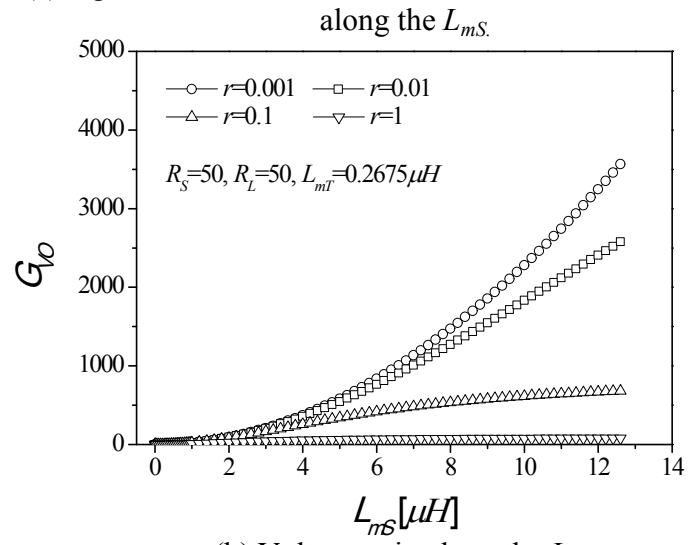

(b) Voltage gain along the $L_{m S}$

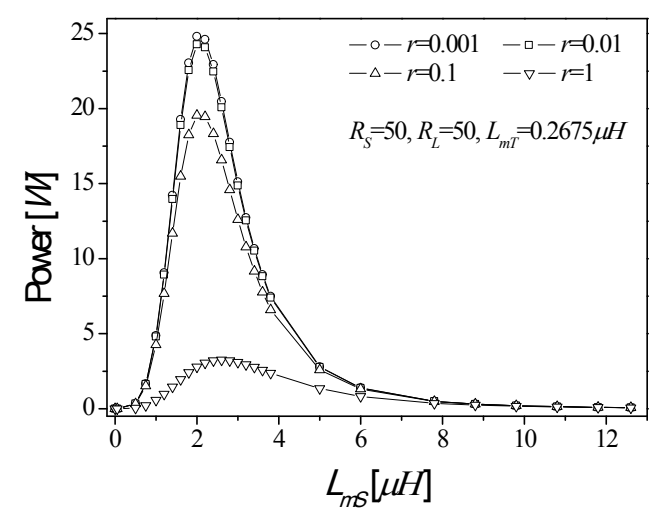

(c) Load power along the $L_{m S}$.

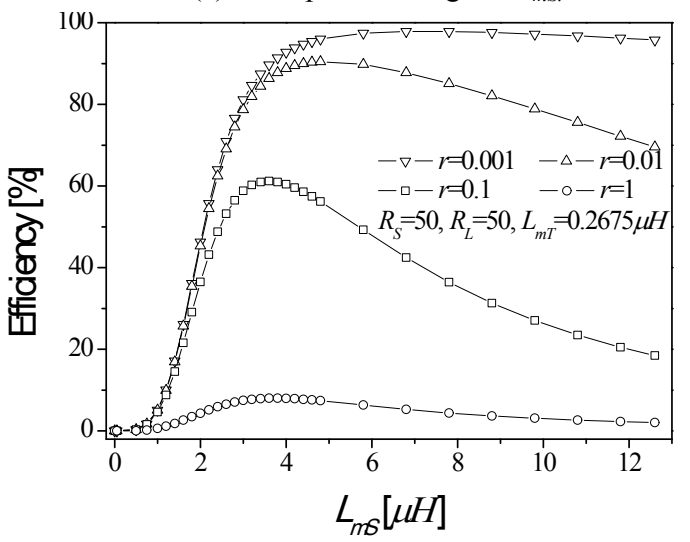

(d) Power efficiency along the $L_{m S}$.

Fig. 8. Simulation results of the proposed explicit model along the $L_{m s}$.
From the experimental results, it can be seen that the theoretical predictions of CMRS are in good agreement with the measurement results, except for some discrepancies. However, these were accounted for as parasitic parameters because the theoretical model considered the mutual coupling between the source coil and the transmitter coil, the transmitter coil and the receiver coil, and the receiver coil and the load coil only, and neglected the other mutual couplings between the coils.

To increase the power and efficiency, an experimental kit using a class-E inverter was newly implemented for an experiment. A DE475-102N21A MOSFET with $V_{D S S}=1000 \mathrm{~V}$ and $I_{D 25}=24 \mathrm{~A}$ was used as a switch in the class-E inverter. A function generator was used as a clock generator since the frequency is easy to change for experimental purposes. The DC power supplied $V_{s}$ of $20 \mathrm{~V}$. The measured self-inductances of Tx and Rx were about $58.1 \mu H$ and $60.3 \mu H$, respectively. The resonant capacitances for each coil were about $1.74 n F$ and $1.68 \mathrm{nF}$. The turn ratio of the source impedance transformer was $4: 1$, and $L_{m s}$ was measured at about $40 \mu \mathrm{H}$. The magnetizing inductance of the load impedance transformer $L_{m L}$ was $40 \mu H$ for $1: 4\left(n_{2}=4\right)$ and the leakage inductances of these two transformers were negligible. The parallel capacitance of the MOSFET was about $1.05 n F$, including both $C_{d s}$ of the MOSFET $0.14 n F$ and $C_{S}=0.91 n F$.

Fig. 11 shows the waveforms of $V_{T}$ and the current of each coil. The phase difference between adjacent coils was $\pi / 2$.

The load power and efficiency were measured for a wide range of values of the load resistor from $10 \Omega$ to $500 \Omega$ as shown in Fig. 12 and Fig. 13. A maximum power of $1.38 \mathrm{~W}$ was achieved as shown in Fig. 12. The highest coil to coil efficiency of $40 \%$ was obtained when $R_{L}=75 \Omega$ and $n_{2}=4$. The highest efficiency was not obtained when $R_{L}=50 \Omega$ as designed because of the difference in $r_{\mathrm{o}}$. The measured $r_{o}$ of the experimental kit was larger than the designed value. It reached almost $6.92 \Omega$ due to the ESR of the lumped capacitors, minor changes of other parameters, and a slight deviation from the perfect resonant condition.

\section{CONCLUSION}

A CMRS was analyzed with closed form equations in detail using the explicit equivalent circuit models proposed in this paper. The maximum power delivery and power efficiency conditions were clearly determined for various design conditions. The phasor relationship for each coil was first fully explained. An optimum CMRS can be designed based on the analytical results, as verified by experiments. A dynamic analysis of the CMRS using phasor transformations [15]-[17] is left for future work, which will complete the analyses of the CMRS. 


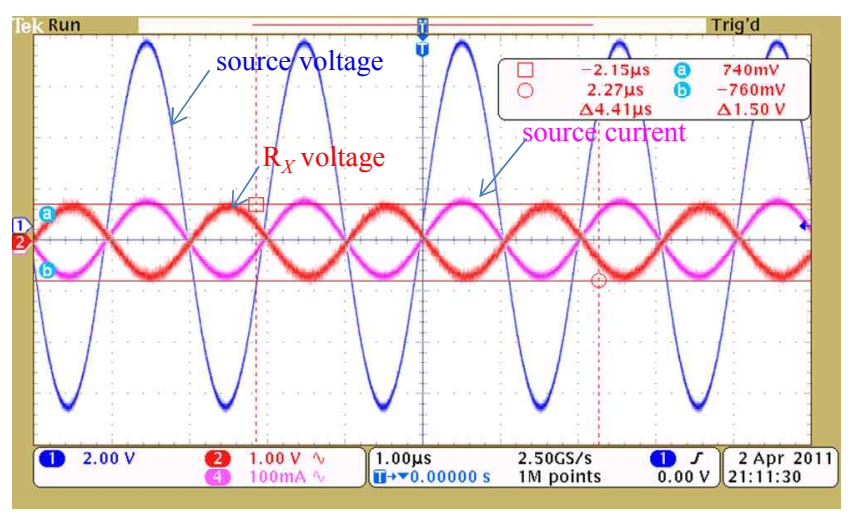

(a) Waveforms of the voltages of the source, load, and receiver coils.

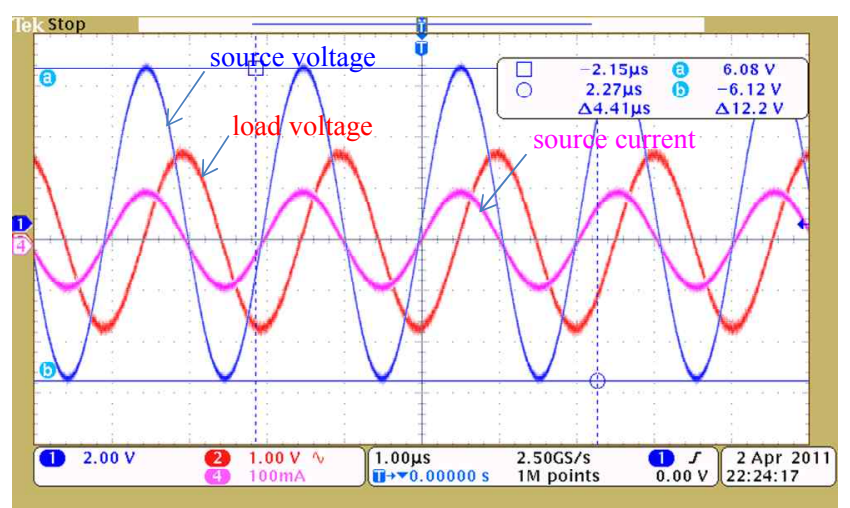

(b) Waveforms of the voltages of the source and load coils, and the current of the source coil when $R_{L}=50 \Omega$.

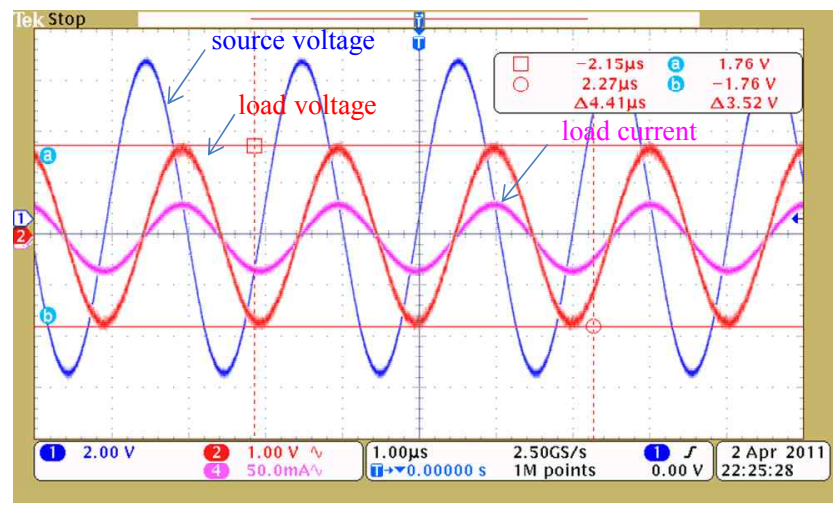

(c) Waveforms of the voltages of the source and load coils, and the current of the load coil when $R_{L}=50 \Omega$.

Fig. 9. The waveforms of the CMRS in fully-resonant condition.

TABLE I

Parameters AND MEASURed VALUeS For EXPERIMENTS

\begin{tabular}{|c|c|c|c|c|c|c|c|}
\hline Parameter & Value & Parameter & Value & Parameter & Value & Parameter & Value \\
\hline$L_{l s}$ & $54.78 \mu H$ & $L_{m s}$ & $2.90 \mu H$ & $r_{c T}$ & $1.34 \Omega$ & $C_{s}$ & $1.803 n F$ \\
\hline$L_{l T}$ & $54.90 \mu H$ & $L_{m T}$ & $0.2675 \mu H$ & $r_{c R}$ & $1.34 \Omega$ & $C_{T}$ & $1.786 n F$ \\
\hline$L_{l R}$ & $54.58 \mu H$ & $L_{m L}$ & $2.87 \mu H$ & $r_{c L}$ & $1.34 \Omega$ & $C_{R}$ & $1.788 n F$ \\
\hline$L_{l L}$ & $55.06 \mu H$ & $R_{S}$ & $50 \Omega$ & $R_{L}$ & $50.92 \Omega$ & $C_{L}$ & $1.784 n F$ \\
\hline
\end{tabular}




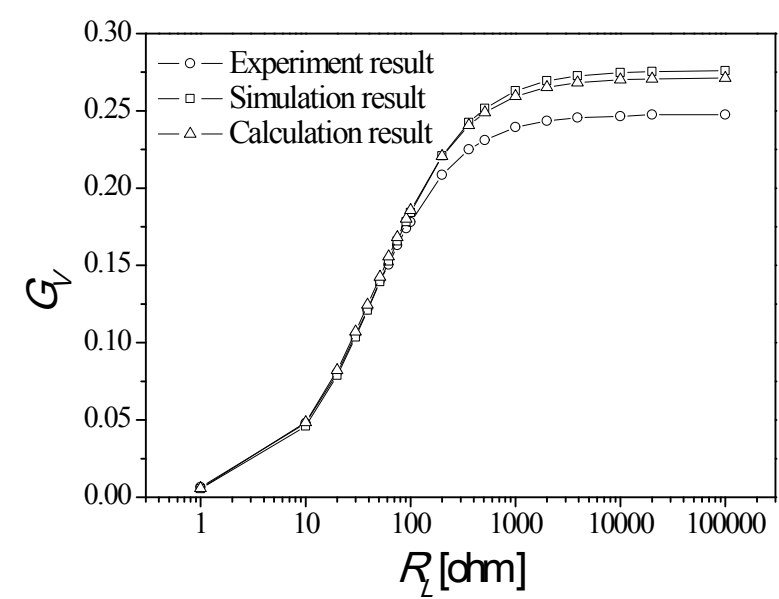

(a) Comparison of the voltage gain along with the load resistance $R_{L}$

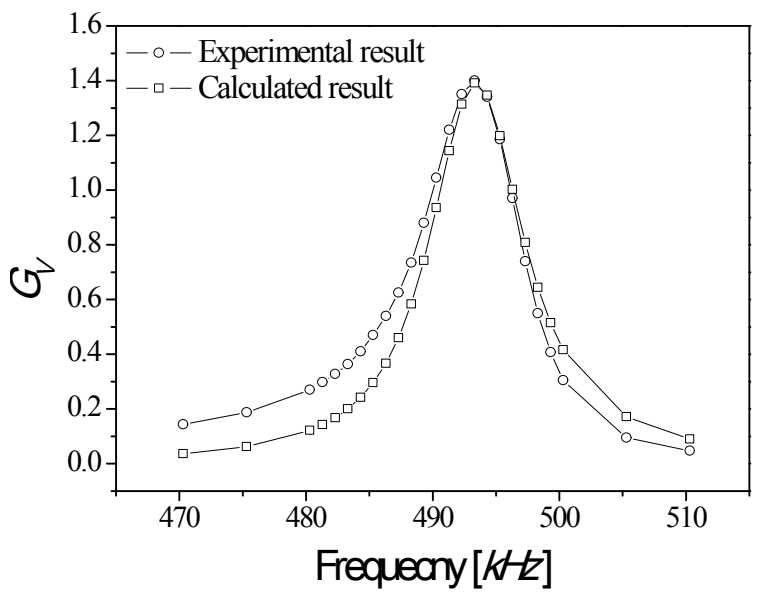

(b) Comparison of the voltage gain along with the frequency.

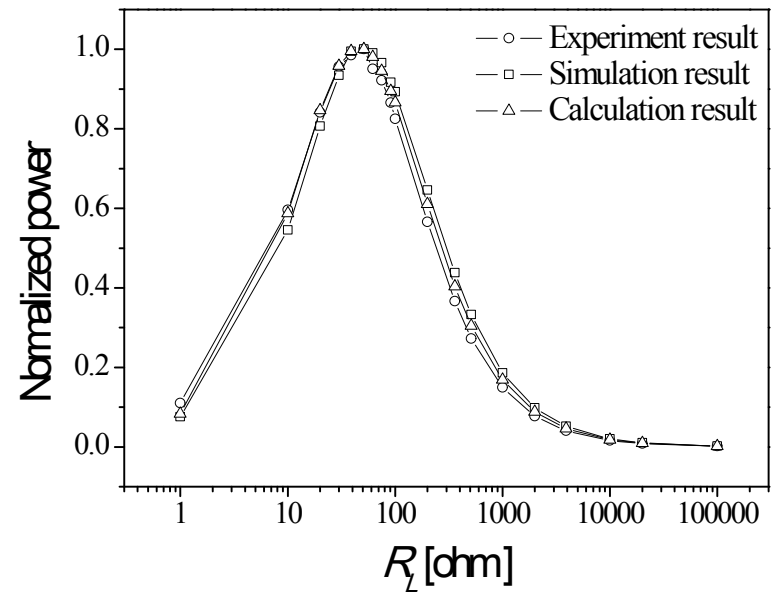

(c) Comparison of the normalized power along with the load resistance $R_{L}$.

Fig. 10. Comparison of the measured data and the calculated data of the CMRS.

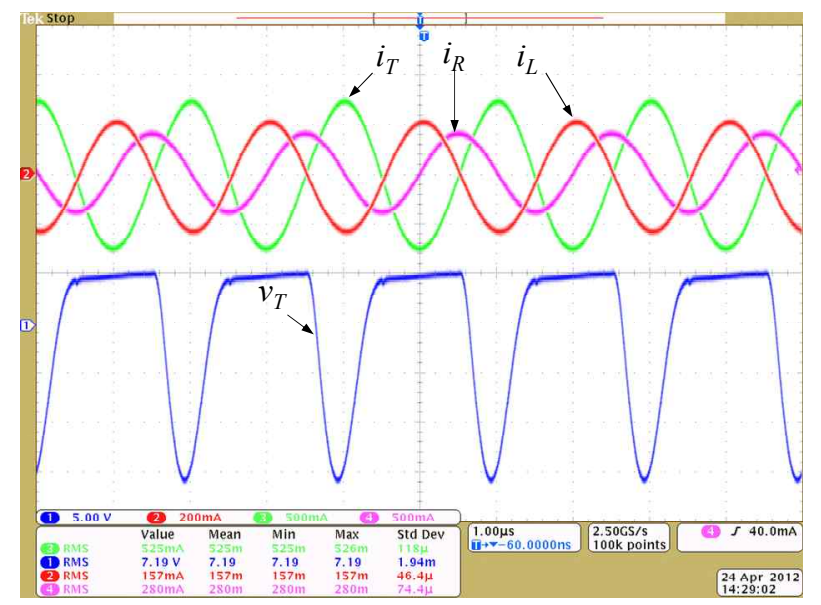

Fig. 11. Secondary voltage of the source transformer $\left(v_{T}\right)$ of the CMRS and each coil current in the resonant mode; the phase difference of each coil current is 90 degree in resonant mode: $V_{s}$ $=20 \mathrm{~V}, f_{\mathrm{s}}=500 \mathrm{kHz}, R_{L}=50 \Omega$,

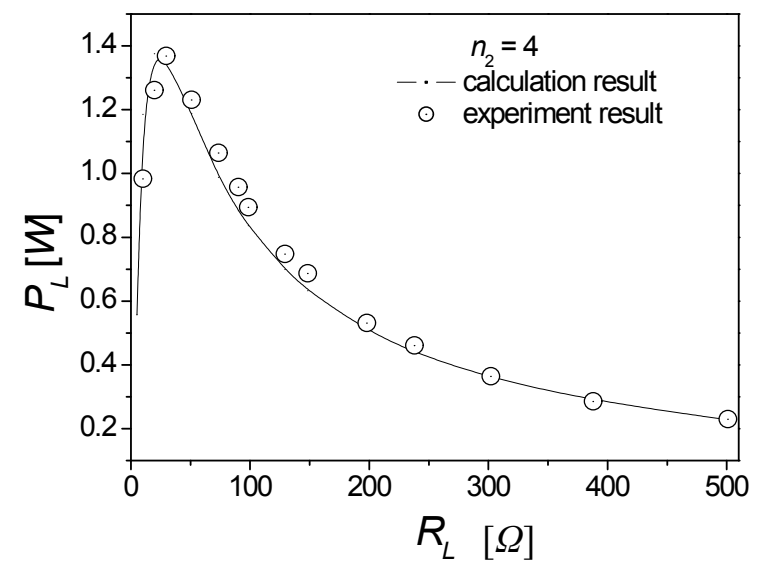

Fig. 12. Comparison of the power between the calculation result and the experiment result vs. $R_{L}: V_{s}=20 \mathrm{~V}, f_{s}=500 \mathrm{kHz}$.

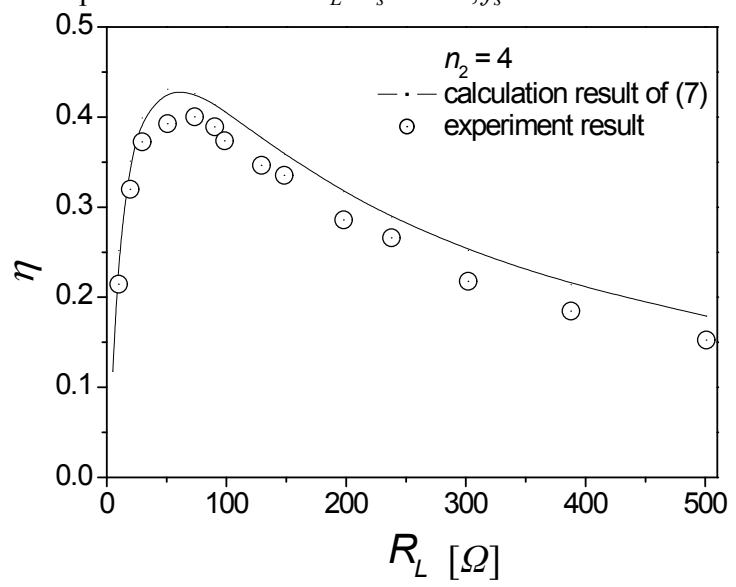

Fig. 13. Comparison of the efficiency between the calculation result and the experiment result vs. $R_{L}: V_{s}=20 \mathrm{~V}, f_{\mathrm{s}}=500 \mathrm{kHz}, R_{L}$ $=50 \Omega$.

\section{ACKNOWLEDGMENT}

This work was supported by the National Research Foundation of Korea (NRF) grant funded by the Korea government (MEST) (No. 2010-0029179) 


\section{REFERENCES}

[1] Y. T. Jang and M. M. Jovanovic, "A contactless electrical energy transmission system for portable-telephone battery chargers," IEEE Trans. Ind. Electron., Vol. 50, pp. 520-527, Jun. 2003.

[2] J. Huh, S. W. Lee, W. Y. Lee, G. H. Cho, and C. T. Rim, "Narrow-width inductive power transfer system for on-line electrical vehicles," IEEE Trans. Power Electron., Vol. 26, No. 12, pp. 3666-3679, Dec. 2011.

[3] N. P. Suh, D. H. Cho, and C. T. Rim, "Design of on-line electric vehicle (olev)," Plenary lecture at the 2010 CIRP Design Conference in Nantes, France, Apr. 2010.

[4] J. Huh, S. W. Lee, C. B. Park, G. H. Cho, and C. T. Rim, "High performance inductive power transfer system with narrow rail width for on-line electric vehicles," in IEEE Energy Conversion Congress and Exposition (ECCE), pp. 647-651, Sep. 2010

[5] S. W. Lee, J. Huh, C. B. Park, N. S. Choi, G. H. Cho, and C. T. Rim, "On-line electric vehicle using inductive power transfer system," in IEEE Energy Conversion Congress and Exposition (ECCE), pp. 1598-1601, Sep. 2010.

[6] J. Huh, W. Y. Lee, G. H. Cho, B. H. Lee, and C. T. Rim, "Characterization of novel inductive power transfer systems for on-line electric vehicles," in IEEE Applied Power Electronics Conference and Exposition (APEC), pp. 1975-1979, Mar. 2011.

[7] S. W. Lee, W. Y. Lee, J. Huh, H. J. Kim, C. B. Park, G. H. Cho, and C. T. Rim, "Active emf cancellation method for i-type pick-up of on-line electric vehicles," in IEEE Applied Power Electronics Conference and Exposition (APEC), pp. 1980-1983, Mar. 2011.

[8] J. Huh and C. T. Rim, "KAIST wireless electric vehicles olev," JSAE Annual Congress, May 2011.

[9] B. H. Lee, H. J. Kim, S. W. Lee, C. B. Park, and C. T. Rim, "Resonant power shoes for humanoid robots," in IEEE Energy Conversion Congress and Exposition (ECCE), pp. 1791-1794, Sep. 2011.

[10] A. Kurs, A. Karalis, R. Moffatt, J. D. Joannopoulos, P. Fisher, and M. Soljacic, "Wireless power transfer via strongly coupled magnetic resonance," Science, Vol. 317, No. 5834, pp. 83-86, Jun. 2007.

[11] A. P. Sample, D. T. Meyer, and J. R. Smith, "Analysis, experimental results, and range adaption of magnetically coupled resonator for Wireless Power Transfer," IEEE Trans. Ind. Electron., Vol. 58, pp. 544-554, Feb. 2011.

[12] Sony Corp., www. sony.net/sonyinfo/news/press/200910/.

[13] Y. H. Kim, S.Y. Kang, S. H. Cheon, M. L. Lee, J. M. Lee, and T. Y. Zyung, "Optimization of wireless power transmission through resonant coupling," International Symposium on Power Electronics Electrical Drivers, Automation and Motion (SPEEDAM), pp. 1069-1073, 2010.

[14] S. H. Lee and R. D. Lorenz, "Development and validation of model for $95 \%$ efficiency, $220 \mathrm{~W}$ wireless power transfer over a $30 \mathrm{~cm}$ air-gap," in IEEE Energy Conversion Congress and Exposition (ECCE), pp. 885-892, Sep. 2010.

[15] C. T. Rim and G. H. Cho, "Phasor transformation and its application to the DC/AC analyses of frequency/phase controlled series resonant converters (SRC)," IEEE Trans. ower Electron., Vol. 5, No. 2, pp. 201-211, Apr. 1990.
[16] C. T. Rim, D. Y. Hu, and G. H. Cho, "Transformers as equivalent circuits for switches: General proofs and D-Q transformation-based analysis," IEEE Trans. Ind. Applicat., pp. 777-785, Jul./Aug. 1990.

[17] C. T. Rim, "Unified general phasor transformation for ac converters," IEEE Trans. Power Electron., Vol. 26, No. 9, pp. 2465-2475, Sep. 2011.

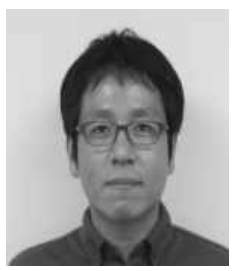

Jin Huh received his B.S. in Electrical Engineering from the Kumoh National Institute of Technology (KIT), Gumi, Korea, in 2002, his M.S. in Electrical Engineering from POSTECH, Pohang, Korea, in 2004, and his Ph.D. in Electrical Engineering from KAIST (Korea Advanced Institute of Technology), Daejeon, Korea, in 2012. He has been involved in developing inductive power transfer systems for on-line electrical vehicles. His current research interests include wireless power transfer, power electronics, and analog circuit design.

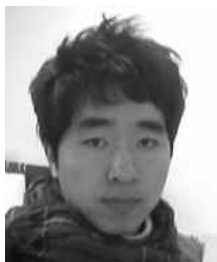

Wooyoung Lee received his B.S. in Electrical Engineering from Ajou University, Suwon, Korea, in 2010 and his M.S. in Electrical Engineering from KAIST, Daejeon, Korea, in 2012. His current research interests include wireless power transfer systems, magnetic field analysis, and power inverters.

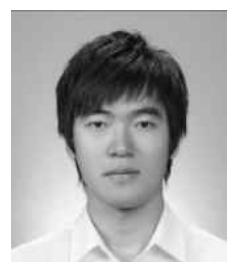

Suyong Choi received his B.S. in Mechanical Engineering from Pusan National University, Pusan, Korea, in 2011 and he is currently pursuing his Ph.D. in Nuclear and Quantum Engineering at KAIST, Daejeon, Korea. His current research interests include wireless power transfer systems used in nuclear power

plants.

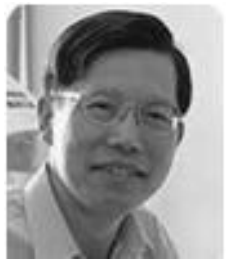

Gyuhyeong Cho was born in Korea in 1953. He received his B.S. from Hanyang University, Seoul, Korea, and his M.S. and $\mathrm{Ph} . \mathrm{D}$. in Electrical Engineering from KAIST, Seoul, Korea, in 1975, 1977, and 1981, respectively. From 1982 to 1983 , he worked for the Westinghouse R\&D Center, PA, USA. He joined the Department of Electrical Engineering at KAIST in 1984 . He has been a tenured Professor since 1991 and was a visiting Professor at the University of Wisconsin, Madison, USA, in 1989. He performed research in the area of power electronics until the late 1990s and has worked on soft switching converters, high power and high voltage inverters, and static VAR compensators. He later shifted to researching analog integrated circuit designs. His current research interests include two main areas. One is the combined area of analog integrated circuits and power electronics, including smart power ICs, such as single-chip power management ICs and Class D audio power amplifiers. The other area is the display drivers ICs for LED, OLED, and LCD flat panel displays. Prof. Cho is a senior member of the IEEE. 


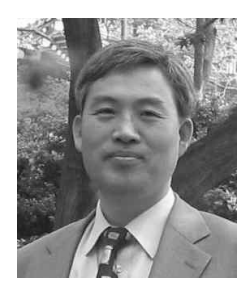

Chuntaek Rim was born in Korea, in 1963.

$\mathrm{He}$ received his B.S. in Electrical

Engineering from the Kumoh Institute of Technology (KIT), Gumi, Korea, in 1985, and his M.S. and Ph.D. in Electrical Engineering from KAIST (Korea Advanced Institute of Technology), Daejeon, Korea, in 1987 and 1990, respectively. Since 2007, he has been an Associate Professor of Nuclear and Quantum Engineering, and an adjunct to Aerospace Engineering in Power Electronics at KAIST. From 1990 to 1995, he was a Military Officer in the Ministry of National Defense in Korea. From 1995 to 2003, he was a Senior Researcher at the Agency for Defense Development, Daejeon, Korea and from 1997 to 1999 he was with Astrium, Portsmouth, U.K. From 2003 to 2007, he was a Senior Director at the Presidential Office, Seoul, Korea. He has been involved in developing Korea's first airborne and spaceborne Synthetic Aperture Radars. He has won 3 prizes from the Korean Government. He is currently developing inductive power transfer systems for on-line electrical vehicles and leading the Mobile Power Electronics Lab, named Tesla Lab, at KAIST. His current research interests include wireless electric vehicles, wireless power systems for robots and bio-medical applications, and energy grid applications. He has authored or coauthored 61 technical papers, written 4 books, and holds more than 40 patents (awarded and pending). 